\title{
Disruption of Glucocorticoid and Mineralocorticoid Receptor-Mediated Responses by Environmental Chemicals
}

\author{
Alex Odermatt* and Christel Gumy
}

\begin{abstract}
Glucocorticoids and mineralocorticoids are key endocrine hormones modulating essential physiological processes such as energy metabolism, cell growth and differentiation, maintenance of blood pressure and immune responses. Despite their importance and the fact that their impaired function has been associated with various diseases, there are only few studies on the potential disruption of glucocorticoid and mineralocorticoid action by xenobiotics. To facilitate the identification and characterization of such chemicals, we established cell-based assays to determine the impact of xenobiotics on different steps of corticosteroid hormone action. Screening of a small library of chemicals led to the identification of several compounds inhibiting the $11 \beta$-hydroxysteroid dehydrogenase (11 $\beta$-HSD) prereceptor enzymes $11 \beta$-HSD1 and/or 11 $\beta$-HSD2 and of chemicals blocking the function of glucocorticoid receptors (GR) and mineralocorticoid receptors (MR). These findings build a basis to extend the search for chemicals acting on additional targets of the corticosteroid hormone pathway and to apply in silico prediction tools in combination with biological testing to screen large numbers of chemicals. The identification of chemicals interfering with corticosteroid action and the elucidation of the underlying molecular mechanisms are relevant with respect to the potential contribution to common diseases such as metabolic syndrome, immune diseases, brain disorders and cancer.
\end{abstract}

Keywords: Cortisol · Endocrine disruptor · Glucocorticoid receptor · Glucocorticoid ·

$11 \beta$-Hydroxysteroid dehydrogenase $\cdot$ Mineralocorticoid receptor $\cdot$ Xenobiotics

\section{Physiological Role of Glucocorticoids and Mineralocorticoids}

Corticosteroid hormones are divided into glucocorticoids (cortisol in humans and corticosterone in rodents) and mineralocorticoids (aldosterone). They are involved in the regulation of many physiological processes, including energy metabolism, electrolyte and blood pressure control, bone metabolism, regulation of brain function,

\footnotetext{
${ }^{*}$ Correspondence: Dr. A. Odermatt Molecular and Systems Toxicology Department of Pharmaceutical Sciences University of Basel

Klingelbergstrasse 50

$\mathrm{CH}-4056$ Basel

Tel.: +41612671530

Fax: +4161267 1515

E-mail: alex.odermatt@unibas.ch
}

cell cycle control, and modulation of stress and inflammatory responses (Fig. 1). ${ }^{[1]}$ An impaired regulation of glucocorticoid action has been associated with various complications including metabolic and cardiovascular diseases, osteoporosis, cataracts, immune diseases, mood and cognitive disorders and cancer. ${ }^{[2-5]}$ Disturbances of mineralocorticoid action have been linked to the occurrence of hypertension and cardiovascular diseases. ${ }^{[6,7]}$ The incidence of these complex diseases increases with increasing age, and in addition to genetic predisposition, factors including life style and the exposure to xenobiotics are likely to play a role in these pathological processes. In developed countries, the exposure to chemicals from the environment may contribute to the high incidence of allergic diseases, cancer and metabolic disturbances. ${ }^{[8-10]}$

\section{Major Targets of the Corticosteroid Hormone Pathway}

Exogenous chemicals can cause disturbances of corticosteroid hormone action at several steps (Fig. 2). Corticosteroids are produced in the adrenal cortex involving several steroidogenic enzymes, and the synthesis of these hormones is tightly regulated by corticotrophin releasing factor (CRF) and adrenocorticotrophic hormone (ACTH) via the hypothalamus-pituitary-adrenal (HPA) axis. Glucocorticoids control their own synthesis by a negative feedback response mediated by inhibition of CRF and ACTH. Upon release into the blood stream, corticosteroid hormones are mainly bound to carrier proteins (transcortin, albumin) and reach cells in peripheral tissues.

At the cellular level, cortisol or corticosterone exert their action through GR and aldosterone acts by activating MR. Importantly, the local activation of the receptors is controlled by two distinct $11 \beta$-HSD prereceptor enzymes. ${ }^{[11]} 11 \beta$-HSD1 is expressed ubiquitously and catalyzes predominantly the reduction of inactive 11-ketoglucocorticoids (cortisone, 11-dehydrocortisone) into active $11 \beta$-hydroxyglucocorticoids (cortisol, corticosterone).[12] This enzyme has a crucial role in potentiating local GR activation in metabolic processes and in the immune system. 


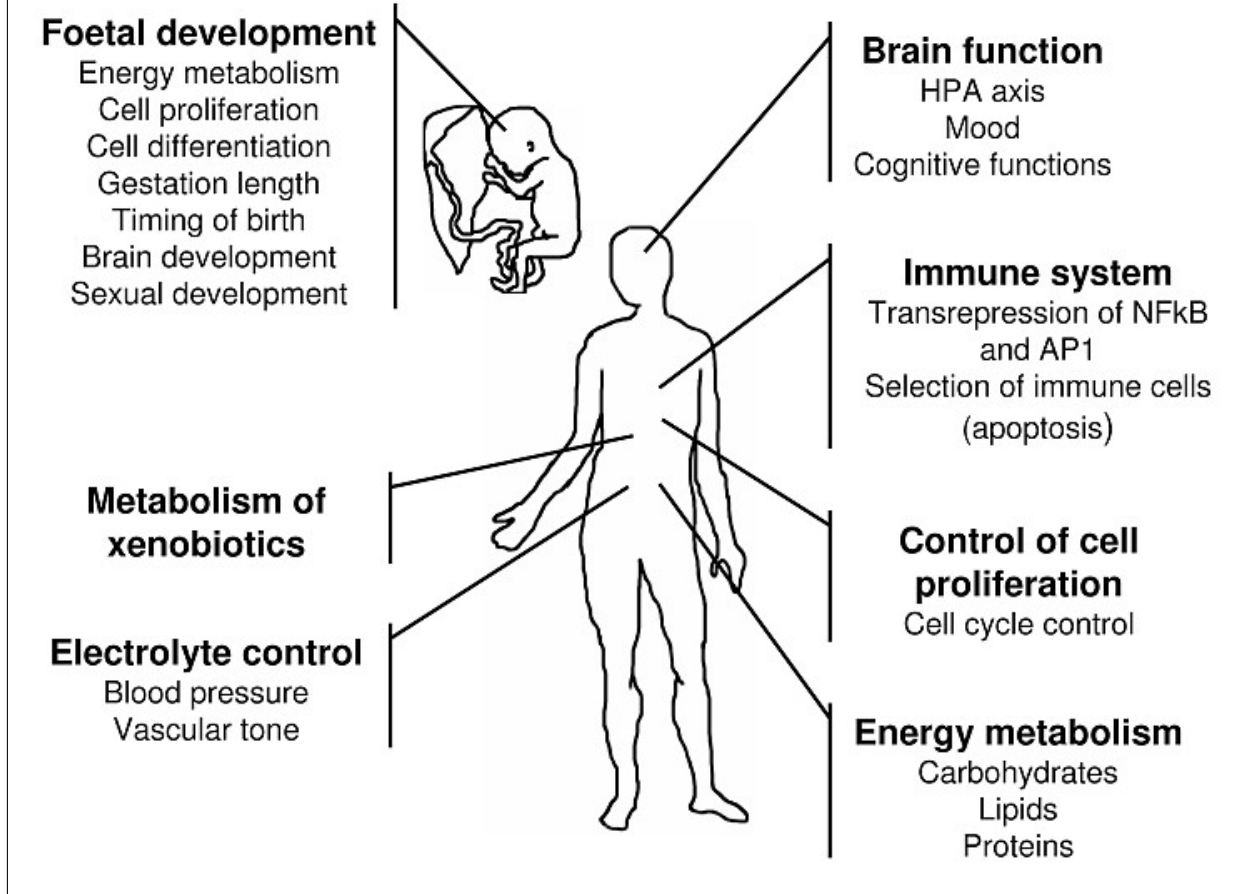

Fig. 1. Overview of physiological functions that are regulated by corticosteroid hormones

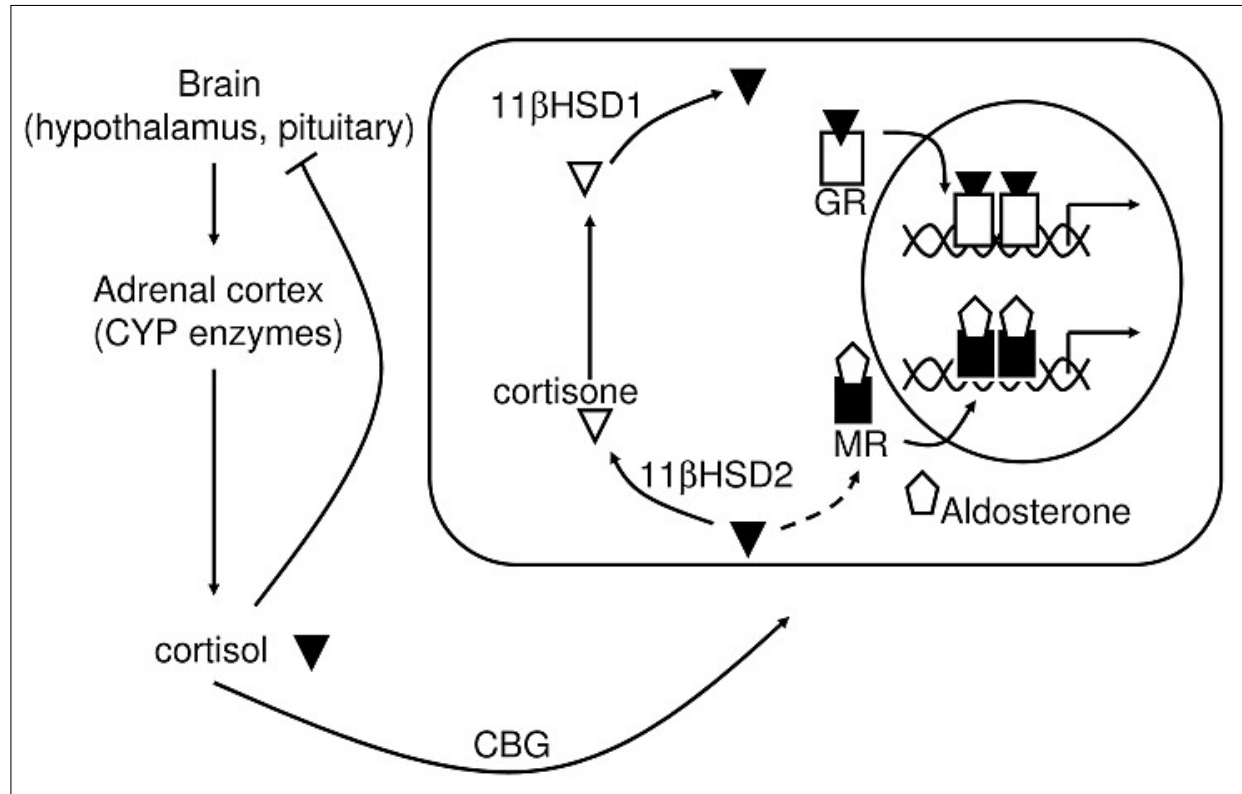

Fig. 2. Schematic overview of targets of corticosteroid hormone action

The second enzyme, 11ß-HSD2, is expressed in cortical collecting ducts and distal tubules in the kidney and in distal colon, where it protects MR from glucocorticoids by converting $11 \beta$-hydroxyglucocorticoids into 11-ketoglucocorticoids. ${ }^{[13]}$ The MR has similar affinities to bind aldosterone and cortisol and circulating concentrations of the latter are 100-1000 times higher, thus, the close proximity of $11 \beta-H S D 2$ to the receptor allows aldosterone to bind. ${ }^{[14]} 11 \beta-\mathrm{HSD} 2$ is also expressed in the syncytiotrophoblast layer of the human placenta, where it pro- tects the fetus from high maternal glucocorticoid concentrations. ${ }^{[15]}$

The tissue-dependent responses upon activation of GR and MR strongly depend on the presence of coactivator and corepressor proteins that interact with the receptor complex, as well as on post-translational modifications of both receptor and associated proteins. Finally, enzymes responsible for the degradation and excretion of the steroid hormones are important to terminate hormone action.

The proteins described above all recognize corticosteroid molecules despite the fact that they belong to different classes of proteins and share very low sequence similarity. This suggests that the binding pockets of these proteins share structural similarity and that the chemicals mimicking corticosteroid molecules might interact with more than one of these proteins. Thus, to assess the potential disruption of corticosteroid hormone action by exogenous chemicals, suitable bioassays are required to measure the activities of the different proteins, with roles in regulation, biosynthesis, transport, intracellular metabolism, receptor and degradation.

\section{Bioassays}

Compared to the extensively studied field of estrogen- and androgen-like actions by environmentally relevant chemicals, there are only few studies focusing on corticosteroid hormones. ${ }^{[16]}$ As a starting point to identify chemicals that act on different steps of glucocorticoid and mineralocorticoid regulation, we established assays to measure the function of human corticosteroid hormone receptors (GR and MR) and glucocorticoid metabolizing enzymes (11 $\beta$-HSD1 and $11 \beta$-HSD2). To distinguish between different steps of receptor activation, the HEK-293 cell line was selected that is devoid of endogenous expression of corticosteroid receptors and hormone metabolizing enzymes.[17] Other cells that are suitable alternatives include COS- 1 and CV-1 cells, ${ }^{[18]}$ but they are not of human origin.

Receptor activity was measured in intact cells transiently expressing recombinant receptor or a green-fluorescence (GFP)chimeric receptor. This allowed the assessment of the effect of a given chemical on ligand binding to the receptor, subsequent translocation of the receptor into the nucleus and receptor-mediated transcriptional activation of a reporter gene. It is important to distinguish the different steps of receptor activation to understand the inhibitory mechanism of a chemical. Spironolactone, as an example, efficiently binds to MR and induces translocation of the receptor into the nucleus. It acts as a partial agonist and therefore antagonizes the potent agonist effect of aldosterone. The bile acid chenodeoxycholic acid inhibits $11 \beta-H S D 2$ and leads to glucocorticoid-induced activation of MR. Although it also induces nuclear translocation of MR in the absence of $11 \beta-H S D 2$, chenodeoxycholic acid neither activates nor antagonizes MR activation by cortisol or aldosterone. ${ }^{[19,20]}$

To assess the effect of chemicals on $11 \beta-H S D$ prereceptor enzyme function, the conversion of cortisone to cortisol or the reverse reaction was measured in lysates or intact HEK-293 cells stably expressing C- 
terminally FLAG-tagged human $11 \beta$-HSD1 or $11 \beta$-HSD2. ${ }^{[21]}$ The impact of $11 \beta$-HSD enzyme inhibition on receptor function was determined in cells cotransfected with $11 \beta$-HSD1 and GR or $11 \beta-H S D 2$ and MR, respectively. As controls for $11 \beta-\mathrm{HSD} 1$ and $11 \beta$-HSD2 the well known inhibitor glycyrrhetinic acid was used, and for MR and GR, spironolactone and RU486 were applied to optimize assay conditions.

The strategy of expressing recombinant corticosteroid receptors in the presence of modulatory proteins to identify chemicals disrupting corticosteroid action by indirect mechanisms will now be extended. The impact on receptor function of chemicals interfering with signaling pathways is currently under investigation. For the efficient analysis of chemicals interfering with corticosteroid hormone action, several cell models stably expressing different combinations of receptor and modulatory protein will be constructed.

\section{Compounds Inhibiting 11 $\beta-H S D 2$}

Liquorice is a well-known endocrine disruptor inducing corticosteroid hormone responses. ${ }^{[22]}$ Glycyrrhetinic acid, which is contained in liquorice, has been identified as a potent inhibitor of $11 \beta$-HSD enzymes. By inhibiting 11ß-HSD2, glycyrrhetinic acid prevents inactivation of cortisol in cortical collecting ducts and distal tubules in the kidney, thereby leading to cortisolinduced MR activation, sodium retention and hypertension. ${ }^{[14,23-26]} 11 \beta-H S D 2$ also has a key function in the regulation of fetal development ${ }^{[15]}$ and protects the fetus from the high maternal cortisol concentrations. The intrauterine exposure to elevated levels of glucocorticoids has been associated with reduced birth weight and glucose intolerance and cardiovascular complications later in life, ${ }^{[27,28]}$ and evidence has been provided that this programming is altered through several generations. ${ }^{[29]}$ This indicates a role for epigenetic factors in the transgenerational information transfer of these alterations. The inhibition of $11 \beta-H S D 2$ during pregnancy by glycyrrhetinic acid or its analogue carbenoxolone leads to elevated glucocorticoid levels and causes reduced birth weight with a higher risk for cardiovascular disease later in life. ${ }^{[25]}$ The inhibition of $11 \beta$-HSD2 by chemicals from the environment in a critical window during pregnancy is thus expected to cause detrimental effects and should be considered as a risk factor for metabolic and cardiovascular diseases.

Grape fruit juice has also been associated with inhibition of $11 \beta$-HSD2, [30,31] and naringenin was suggested as the active compound. However, naringenin is a weak $11 \beta$-HSD2 inhibitor $\left(\mathrm{IC}_{50}>300 \mu \mathrm{M}\right),{ }^{[21,32]}$ and it is more likely that other, as yet un- identified, compounds are responsible for inhibition of $11 \beta-H S D 2$. Additional compounds of the triterpenoid and flavonoid class of chemicals that inhibited $11 \beta$-HSD2 include abietic acid, gossypol, magnolol and tea polyphenols. However, their relatively weak activities observed in intact cell assays suggest that these compounds are unlikely to cause physiological consequences by inhibiting $11 \beta$-HSD2.[33]

We then tested about 100 environmentally relevant chemicals that were selected based on evidence in the literature for potential interference with corticosteroid action, for their effect on $11 \beta$-HSD2 activity. In addition to the compounds mentioned above, abietic acid, fusidic acid, zearalenone, 4-tert-octylphenol, 4-nonylphenol, bisphenol A, endosulfan and several dithiocarbamates and organotins inhibited 11 $\beta$-HSD2. ${ }^{21]}$ Among these, abietic acid, bisphenol A, dithiocarbamates and organotins inhibited $11 \beta-H S D 2$ at subcytotoxic concentrations. Dithiocarbamates and organotins were studied in more detail.

Dithiocarbamate chemicals are widely used in agriculture as pesticides or fungicides and as vulcanization accelerators in latex production (mainly in gloves). Dithiocarbamates are considered to be responsible for the allergic reactions against rubber products. ${ }^{[34]}$ Interestingly, disulfiram, known as Antabus to treat alcoholic patients, was also active. Among the dithiocarbamates analyzed, disulfiram and thiram were most potent with an $\mathrm{IC}_{50}$ value of $130 \mathrm{nM}$, followed by maneb, diethyldithiocarbamate and zineb. ${ }^{[35]}$ The inhibitory potential of disulfiram and thiram to inhibit 11 $\beta$-HSD2 was comparable with that for aldehyde dehydrogenase. These chemicals irreversibly inhibited $11 \beta$-HSD2, probably by covalent carbamoylation of catalytically important cysteine residues. Glutathione protected $11 \beta$-HSD2 from inhibition by dithiocarbamates, suggesting that these chemicals are most critical in situations of oxidative stress, when intracellular glutathione concentrations are low. The exposure to the dithiocarbamates maneb and zineb has been associated with acute renal failure and nephrotic syndrome in agricultural workers, as well as kidney damage and reduced body weights in the offspring from exposed pregnant rats. The inhibition of $11 \beta$-HSD2 may contribute to some of the observed toxic effects of these chemicals in kidney and on blood pressure, as well as in placenta and on fetal development.

We identified several organotins including trialkyltins and dialkyltins that potently inhibited $11 \beta$-HSD2 but not $11 \beta$-HSD1, $17 \beta$-HSD1 or $17 \beta$-HSD2. ${ }^{[36]}$ They reversibly inhibited the enzyme with comparable potencies in assays with cell lysates or intact cells. Analysis of the inhibitory mechanism suggested that organotins interfere with $11 \beta-H S D 2$ function by modification of cysteine residues. Dithiothreitol, but not glutathione, protected from organotindependent inhibition. Enhanced glucocorticoid concentrations, due to disruption of $11 \beta$-HSD2 function, may contribute to the observed organotin-dependent toxicity in some glucocorticoid-sensitive tissues such as thymus and placenta. Reduced birth weight and thymus involution, observed upon exposure to organotins, can also be caused by excessive glucocorticoid levels.

\section{Chemicals Disrupting MR Activation}

The same set of about 100 chemicals was screened using the MR nuclear translocation assay to test them for agonist or antagonist properties. Aldosterone-mediated nuclear translocation of MR was inhibited by various chemicals with different properties, including bisphenol A, endosulfan, 4-nonylphenol, vinclozolin, zearalenone, and some phthalate derivatives. All of these chemicals inhibited nuclear translocation of MR at high concentrations of 20-50 $\mu \mathrm{M}$ that are unlikely to be physiologically relevant. The screening of this small number of chemicals showed that chemicals interfering with MR activation exist, although none of the identified compounds was of high potency. It cannot be excluded at this point that some of the identified chemicals have more pronounced effects under certain conditions such as oxidative stress or upon preincubation for a prolonged time. Clearly, a more extensive screening will be necessary for the identification of chemicals with physiologically relevant effects.

The contribution of dithiocarbamatedependent $11 \beta$-HSD2 inhibition to cortisol-induced activation of corticosteroid receptors is unclear, since we found that high concentrations of these chemicals also could block MR and GR. This is in line with a recent study by Garbrecht et al. who described thiram-dependent inhibition of GR activation. ${ }^{[37]}$ An inhibition of $11 \beta-H S D 2$ and/or MR and GR is only likely to occur under conditions of glutathione depletion, and further studies are required to see which protein is inhibited at the lowest concentrations.

\section{Compounds Inhibiting 11 $\beta$-HSD1}

Inhibition of 11 $\beta$-HSD1-dependent glucocorticoid activation is currently considered as a promising strategy to treat patients with metabolic syndrome ${ }^{[5]}$ and many medium and large pharmaceutical companies have inhibitor development programs. While preventing excess production of active glucocorticoids is important to avoid 
adverse metabolic effects and inhibition of $11 \beta$-HSD1 is beneficial in such situations, glucocorticoid deficiency has been associated with impaired immune responses in inflammatory reactions ${ }^{[38]}$ and a reduced $11 \beta$-HSD1 expression has been found in some forms of cancer. ${ }^{[39,40]}$ Thus, depending on the tissue and on the metabolic state, inhibition of $11 \beta$-HSD1 may have different consequences.

Several compounds inhibiting $11 \beta$ HSD1 were identified upon screening our small library. Zearalenone, 4-tertoctylphenol, 4-nonylphenol, methyljasmonate, dibenzoylmethane and 2,2'-dihydroxybiphenyl were weak inhibitors, unlikely to be of physiological relevance. ${ }^{[21]}$ Abietic acid and flavanone as well as some monohydroxylated flavanone derivatives were more potent. While abietic acid displayed a similar activity as the one observed with glycyrrhetinic acid and carbenoxolone and inhibited both 11ß-HSD1 and $11 \beta$-HSD2, flavanone and its derivatives selectively inhibited $11 \beta$-HSD1 by competing with substrate binding. Analysis of the structural requirements for the inhibitory effect of flavanones revealed that hydroxylation at position 6 leads to reduced potency. Multiple hydroxylated flavanones such as the trihydroxylated naringenin also showed weak inhibitory activities on $11 \beta$-HSD enzymes. In addition, the inhibitory effect was lost in flavones, which have a double bond between atoms $\mathrm{C} 2$ and $\mathrm{C} 3$. In a recent study, we provided evidence that coffee contains compounds inhibiting $11 \beta$-HSD1, which might explain in part the anti-diabetic effect associated with regular coffee consumption. ${ }^{[41]}$ Thus, some natural compounds may contribute to the positive health effects of fruits and vegetables by inhibiting $11 \beta$-HSD1 and they might be used as food supplements.

$11 \beta-H S D 1$ is a relatively unspecific enzyme and accepts several substrates. Several reports suggested a role for $11 \beta$-HSD1 in phase I detoxification of carbonyl group containing xenobiotics. ${ }^{[42,43]}$ In addition, several studies recently described an alternative role of $11 \beta$-HSD1 in the metabolism of 7-oxygenated cholesterol metabolites. [44-47] In intact cells, 11 $\beta$-HSD1 catalyzed exclusively the conversion of 7-ketocholesterol to 7 $\beta$-hydroxycholesterol.[44-48] 7-Ketocholesterol is a major oxidized cholesterol metabolite and is formed mainly upon processing cholesterol-rich food. It is found at micromolar concentrations in cataract lenses and in atherosclerotic plaques. The role of $11 \beta$-HSD1 in these pathological conditions and the impact of its inhibition remains to be investigated. Nevertheless, an accumulation of orally administered 7-ketocholesterol was observed in rats treated with the inhibitor carbenoxolone. ${ }^{[44]}$ More recently, we found that $11 \beta$-HSD1 metabolizes 7-ketodehydroepiandrosterone and 7-ketopregnenolone, suggesting a role not only in the detoxification of oxidized cholesterol from food but also of oxidized steroid hormone metabolites. ${ }^{[49]}$

\section{Interference of Chemicals with GR Function}

The same set of about 100 chemicals was screened using our nuclear GR translocation assay. Bisphenol A, zearalenone and some phthalate derivatives inhibited cortisol-induced nuclear translocation of GR. These chemicals also inhibited nuclear MR translocation. Bisphenol A and zearalenone inhibited the function of all four proteins tested, suggesting either an unspecific mechanism or that these chemicals mimic the steroid hormone cortisol and are therefore recognized by all four proteins. In addition, dibenzoylmethane and abietic acid at concentrations of 20-50 $\mu \mathrm{M}$ were shown to inhibit GR translocation. Due to the high concentrations required for inhibition, a direct effect on GR was considered not physiologically relevant. In these experiments, effects on transcriptional expression or on protein stability after prolonged incubation with the chemical of interest have not been assessed.

In a recent study, we found that the organotin dibutyltin inhibits the activation of GR at submicromolar concentrations (unpublished observations). An inhibition of GR function by dibutyltin might explain some of the immunotoxic effects and effects on energy metabolism of this chemical, with relevance to chronic inflammatory disorders as well as metabolic diseases.

\section{Outlook}

Screening of a very small library of environmentally relevant chemicals with cell-based assays for GR, MR, 11 $\beta$-HSD1 and $11 \beta$-HSD2 led to the identification of several compounds that interfere with the function of these proteins, emphasizing the importance to consider potential interferences with glucocorticoid and mineralocorticoid hormone action for the safety assessment of chemicals. Disruption of corticosteroid hormone action by xenobiotics might contribute to metabolic and cardiovascular diseases, impaired brain function, developmental disorders, immune diseases and cancer. To assess the safety of chemicals and avoid interferences with corticosteroid hormone action, the development of additional biological in vitro and in vivo tests is required for the detection of disturbances at various levels of hormone action, including HPA axis regulation, steroidogenesis, transport protein activity, activities of metabolizing enzymes and receptor function. The development of suitable in silico prediction tools and systemic approaches, including analyses of effects of xenobiotics on the transcriptome and proteome, is necessary for the identification of chemicals disrupting corticosteroid hormone action and to understand their mechanisms of action.

\section{Acknowledgements}

This work was supported by grants from the Swiss National Science Foundation No 310000-112279 and NRP50 'Endocrine Disruptors' No 4050-066575. A.O. is a Novartis Research Foundation Professor.

Received: March 22, 2008

[1] R. M. Sapolsky, L. M. Romero, A. U. Munck, Endocr. Rev. 2000, 21, 55

[2] M. C. Carnahan, D. A. Goldstein, Curr Opin. Ophthalmol. 2000, 11, 478.

[3] J. K. Belanoff, K. Gross, A. Yager, A. F. Schatzberg, J. Psychiatr. Res. 2001, 35, 127.

[4] J. R. Seckl, Eur. J. Endocrinol. 2004, 151 Suppl 3, U49.

[5] A. G. Atanasov, A. Odermatt, Endocr Metab. Immune Disord. Drug Targets 2007, 7, 125.

[6] R. Rocha, J. W. Funder, Ann. N. Y. Acad. Sci. 2002, 970, 89.

[7] F. J. Frey, A. Odermatt, B. M. Frey, Curr. Opin. Nephrol. Hypertens. 2004, 13, 451.

[8] E. M. Vivian, Curr. Med. Res. Opin. 2006, 22, 297.

[9] F. Grun, B. Blumberg, Rev. Endocr. Metab. Disord. 2007, 8, 161.

[10] W. Eder, M. J. Ege, E. von Mutius, New Engl. J. Med. 2006, 355, 2226.

[11] P. M. Stewart, Z. S. Krozowski, Vitam. Horm. 1999, 57, 249.

[12] A. Odermatt, Curr. Enzyme Inhib. 2005, 1, 97.

[13] P. C. White, T. Mune, A. K. Agarwal, Endocr. Rev. 1997, 18, 135

[14] A. Odermatt, P. Arnold, F. J. Frey, J. Biol. Chem. 2001, 276, 28484.

[15] P. J. Burton, B. J. Waddell, Biol. Reprod. 1999, 60, 234

[16] A. Odermatt, C. Gumy, A. G. Atanasov, A. A. Dzyakanchuk, J. Steroid Biochem. Mol. Biol. 2006, 102, 222.

[17] A. Odermatt, P. Arnold, A. Stauffer, B. M. Frey, F. J. Frey, J. Biol. Chem. 1999, 274 , 28762.

[18] A. G. Rebuffat, S. Tam, A. R. Nawrocki, M. E. Baker, B. M. Frey, F. J. Frey, A. Odermatt, Mol. Cell Endocrinol. 2004, 214, 27.

[19] C. Quattropani, B. Vogt, A. Odermatt, B. Dick, B. M. Frey, F. J. Frey, J. Clin. Invest. 2001, 108, 1299.

[20] A. T. Stauffer, M. K. Rochat, B. Dick, F. J. Frey, A. Odermatt, J. Biol. Chem. 2002 , 277, 26286.

[21] R. A. Schweizer, A. G. Atanasov, B. M. Frey, A. Odermatt, Mol. Cell Endocrinol. 2003, 212, 41 .

[22] J. A. Molhuysen, J. Gerbrandy, L. A. De Vries, J. C. De Jong, J. B. Lenstra, K. P. 
Turner, J. G. G. Borst, Lancet 1950, 259, 381.

[23] E. P. Gomez-Sanchez, C. E. GomezSanchez, Am. J. Physiol. 1992, 263, E1125.

[24] R. S. Lindsay, R. M. Lindsay, C. R. Edwards, J. R. Seckl, Hypertension 1996, 27, 1200.

[25] R. S. Lindsay, R. M. Lindsay, B. J. Waddell, J. R. Seckl, Diabetologia 1996, 39, 1299.

[26] P. Ferrari, A. Sansonnens, B. Dick, F. J. Frey, Hypertension 2001, 38, 1330.

[27] R. S. Goland, S. Jozak, W. B. Warren, I. M. Conwell, R. I. Stark, P. J. Tropper, J. Clin. Endocrinol. Metab. 1993, 77, 1174.

[28] A. L. Fowden, Reprod. Fertil. Dev. 1995, 7, 351 .

[29] A. J. Drake, B. R. Walker, J. R. Seckl, Am. J. Physiol. Regul. Integr. Comp. Physiol. 2005, 288, R34.

[30] Y. S. Lee, B. J. Lorenzo, T. Koufis, M. M. Reidenberg, Clin. Pharmacol. Ther. 1996, $59,62$.

[31] M. Palermo, D. Armanini, G. Delitala, Clin. Endocrinol. (Oxf) 2003, 59, 143.

[32] Y. D. Zhang, B. Lorenzo, M. M. Reidenberg, J. Steroid. Biochem. Mol. Biol. 1994, 49, 81.

[33] A. Odermatt, Swiss Med. Wkly 2004, 134, 4.

[34] E. Nettis, G. Assennato, A. Ferrannini, A. Tursi, Clin. Exp. Allergy 2002, 32, 441.

[35] A. G. Atanasov, S. Tam, J. M. Rocken, M. E. Baker, A. Odermatt, Biochem. Biophys. Res. Commun. 2003, 308, 257.

[36] A. G. Atanasov, L. G. Nashev, S. Tam, M. E. Baker, A. Odermatt, Environ. Health Perspect. 2005, 113, 1600.

[37] M. R. Garbrecht, Z. S. Krozowski, J. M. Snyder, T. J. Schmidt, Steroids 2006, 71, 895.
[38] M. Schmidt, C. Weidler, H. Naumann, S. Anders, J. Scholmerich, R. H. Straub, Arthritis Rheum. 2005, 52, 1711.

[39] C. Finckh, A. Atalla, G. Nagel, B. Stinner, E. Maser, Chem. Biol. Interact. 2001, 130, 761.

[40] S. Gronau, D. Koenig Greger, M. Jerg, H. Riechelmann, Clin. Otolaryngol. 2002 , 27, 453.

[41] A. G. Atanasov, A. A. Dzyakanchuk, R. A. Schweizer, L. G. Nashev, E. M. Maurer, A. Odermatt, FEBS Lett. 2006, 580, 4081.

[42] E. Maser, G. Bannenberg, Biochem Pharmacol. 1994, 47, 1805.

[43] M. Hult, C. S. Nobel, L. Abrahmsen, D. A. Nicoll-Griffith, H. Jornvall, U. C. Oppermann, Chem. Biol. Interact. 2001 $130,805$.

[44] R. A. Schweizer, M. Zurcher, Z. Balazs, B. Dick, A. Odermatt, J. Biol. Chem. 2004, 279, 18415 .

[45] M.Hult, B.Elleby, N. Shafqat, S. Svensson, A. Rane, H. Jornvall, L. Abrahmsen, U. Oppermann, Cell Mol. Life Sci. 2004, 61 , 992.

[46] H. Shiotsuki, Y. Maeda, K. Chijiiwa, J. Steroid Biochem. Mol. Biol. 2004, 91, 185.

[47] W. Song, J. Chen, W. L. Dean, R. N. Redinger, R. A. Prough, J. Biol. Chem. 1998, 273, 16223.

[48] C. Frick, A. G. Atanasov, P. Arnold, J. Ozols, A. Odermatt, J. Biol. Chem. 2004, 279,31131

[49] L. G. Nashev, C. Chandsawangbhuwana, Z. Balazs, A. G. Atanasov, B. Dick, F. J. Frey, M. E. Baker, A. Odermatt, PLoS ONE 2007, 2, e561. 